\title{
ON A DECOMPOSITION OF A QUATERNARY QUADRATIC FORM*
}

\author{
BY G. Y. RAINICH
}

For a binary quadratic form it is very easy to prove that it can be decomposed in a unique way into a sum of a form of the type $e\left(x^{2}+y^{2}\right)$ and another which by an orthogonal transformation can be reduced to $q\left(x^{2}-y^{2}\right)$. $\dagger$ It is the purpose of this note to prove an analogous theorem for quadratic quaternary forms, namely, that such a form can be presented as the sum of three forms:

(a) a form of the type $e\left(x^{2}+y^{2}+z^{2}+t^{2}\right)$,

(b) a form which by an orthogonal transformation can be reduced to $q\left(x^{2}+y^{2}-z^{2}-t^{2}\right)$, and

(c) a square of a linear form $(a x+b y+c z+d t)^{2}$.

The decomposition in general is unique; an exceptional case is indicated below.

It seems that the decomposition is interesting in itself but it gains in interest because of possible application in physics. Translated into tensor language the theorem may be stated as follows: a symmetric tensor of rank two in four-dimensional space may be presented, in general in a unique way, as the sum of a tensor of the type of a hydrodynamical tensor $\delta g_{i j}+\rho u_{i} u_{i}$, where $\delta$ corresponds to pressure, $\rho$ to density and $u_{i}$ is a unit vector, the four-dimensional velocity; and an electromagnetic stress-energy tensor. $\ddagger$ If the theorem were true in pseudoeuclidean space, it would mean that the contracted Riemann tensor, set equal to the sum of the two above tensors, determines (in general, uniquely) the field of matter and the electromagnetic field. However, the situation in pseudo-euclidean space is more complicated and the theorem there is not true without modifica-

* Presented to the Society, August 27, 1929.

$\dagger$ These two types correspond, if the forms are interpreted as second differential forms of surfaces, to a sphere and to a minimal surface respectively.

$\ddagger$ For a proof that this tensor is of the type (b), see Proceedings of the National Academy of Science, vol. 10 (1924), p. 126; or Transactions of this Society, vol. 27 (1925), p. 117. 
tion and additional restrictions which lie in the nature of things; we limit ourselves here to the euclidean case and leave the applications aside, reserving the treatment of the pseudoeuclidean case for another publication.*

By an orthogonal transformation every given form can be reduced to one of the type

$$
f x^{2}+g y^{2}+h z^{2}+k t^{2} .
$$

The problem can be stated then in the following way: is it possible to subtract from (1) an expression of the type (a) and an expression of the type (c) so that the remainder should be reducible by an orthogonal transformation to the type (b)? It is convenient, instead of quadratic forms to consider corresponding matrices. Without writing out separately the matrices corresponding to (1), (a), and (c), we write at once the matrix corresponding to the first minus the other two; it is:

$$
\left\|\begin{array}{cccc}
f-a^{2}-e & -a b & -a c & -a d \\
-b a & g-b^{2}-e & -b c & -b d \\
-c a & -c b & h-c^{2}-e & -c d \\
-d a & -d b & -d c & k-d^{2}-e
\end{array}\right\|
$$

This matrix must be transformable by an orthogonal transformation into one of the type

$$
\left\|\begin{array}{rrrr}
q & 0 & 0 & 0 \\
0 & q & 0 & 0 \\
0 & 0 & -q & 0 \\
0 & 0 & 0 & -q
\end{array}\right\|
$$

The square of this matrix is $q^{2}$ times the unit matrix. But an orthogonal transformation brings a unit matrix into a unit matrix; therefore the square of the matrix (2) must be a unit matrix multiplied by a number, and the elements outside of the main diagonal of this matrix (the square of (2)) must necessarily be zero; we obtain thus six equations:

* Compare L. Hanni, Verwendung des Energie-Impulstensors, etc., Tôhoku Mathematical Journal, vol. 27 (1926), pp. 277-292. 
1930.]

$$
\begin{aligned}
& a b\left(a^{2}+b^{2}+c^{2}+d^{2}+2 e-f-g\right)=0, \\
& a c\left(a^{2}+b^{2}+c^{2}+d^{2}+2 e-f-h\right)=0, \\
& a d\left(a^{2}+b^{2}+c^{2}+d^{2}+2 e-f-k\right)=0, \\
& b c\left(a^{2}+b^{2}+c^{2}+d^{2}+2 e-g-h\right)=0, \\
& b d\left(a^{2}+b^{2}+c^{2}+d^{2}+2 e-g-k\right)=0, \\
& c d\left(a^{2}+b^{2}+c^{2}+d^{2}+2 e-h-k\right)=0 .
\end{aligned}
$$

We could satisfy these equations by making three of the four numbers $a, b, c, d$ zero, for example, $a=b=c=0$; then the matrix (2) becomes a diagonal matrix without any transformation, but in order that it should be possible to make it assume the form (3) by the choice of the two numbers $e$ and $d$ still at our disposal, two of the given numbers $f, g, h$ must be equal. Unless two of the numbers $f, g, h, k$ are equal we will not achieve our purpose by making three of the numbers $a, b, c, d$ vanish. We may try to make two of these numbers zero, for example,

$$
a=b=0 \text {. }
$$

This satisfies the first five of the equations (4) and to satisfy the last one we have to make

$$
c^{2}+d^{2}+2 e=h+k \text {. }
$$

The matrix (6) becomes now

$$
\left\|\begin{array}{cccc}
f-e & 0 & 0 & 0 \\
0 & g-e & 0 & 0 \\
0 & 0 & h-c^{2}-e & -c d \\
0 & 0 & -d c & k-d^{2}-e
\end{array}\right\| .
$$

An orthogonal transformation of the variables $z$ and $t$ only is required to transform this into a diagonal matrix, and any additional orthogonal transformations that would not destroy diagonality would not affect the elements $f-e$ and $g-e$. The other two elements of the resulting diagonal matrix are the roots of the quadratic equation

$$
x^{2}-\left(h-c^{2}-e+k-d^{2}-e\right) x+\left|\begin{array}{cc}
h-c^{2}-e & -c d \\
-d c & k-d^{2}-e
\end{array}\right|=0
$$


the coefficient of $x$ in this equation is zero in view of (6) so that these two elements must be identified with $q$ and $-q$ of the matrix (3), and therefore the elements $f-e$ and $g-e$ also must be two numbers whose sum is zero; we obtain in this way $f-e+g-e=0$, or

$$
e=\frac{1}{2}(f+g) .
$$

It only remains for us to arrange it so that the two opposite numbers $f-e$ and $g-e$ be of the same absolute value as the roots of the quadratic equation above, that is, that the products of these two pairs be the same, if we want to have a matrix of the form (3). We have thus the equation

$$
\left(h-c^{2}-e\right)\left(k-d^{2}-e\right)-c^{2} d^{2}=(f-e)(g-e)
$$

which in view of (6) and (8) becomes

$$
k c^{2}+h d^{2}=h k-f g .
$$

Eliminating $e$ from (6) and (8) we obtain also

$$
c^{2}+d^{2}=h+k-(f+g) .
$$

The last two equations can be easily solved for $c^{2}$ and $d^{2}$, if we assume that $h \neq k$, and give

$$
d^{2}=\frac{(h-f)(h-g)}{h-k}, \quad c^{2}=\frac{(k-f)(k-g)}{k-h} .
$$

This furnishes real values for $c$ and $d$ only if the fractions in the right hand sides of (11) are both positive; but then we have a choice of six possibilities as to which two of the four numbers $a, b, c, d$ we set equal to zero and among these six possibilities there is always one and only one which gives real values for the remaining two. To see this it is enough to notice that for $d$, for example, to be real it is necessary and sufficient that the corresponding number $h$ be such that

$$
(h-f)(h-g)(h-k)
$$

be positive; this means that $h$ must be either the greatest or the second smallest among the numbers $f, g, h, k$. We must therefore arrange our notations in such a way that $h$ and $k$ be the greatest and second smallest among the numbers $e, f, g, h$; then 
the formulas (11), (8), and (5) furnish us a solution of the problem.

We still have to consider the possibility of satisfying the equations (4) by making vanish more than one of the expressions in brackets in the left hand sides of these equations and less than two of the numbers $a, b, c, d$; but it is easy to see that this is possible only in the exceptional case mentioned above, namely, where two of the numbers $f, g, h, k$ are equal. Barring this exceptional case we have then a unique system of values for $e, a^{2}, b^{2}, c^{2}$, and $d^{2}$; but we can choose the signs of $c$ and $d$.

The University of Michigan

\title{
ON THE PHRAGMÉN-BROUWER THEOREM*
}

\author{
BY W. A. WILSON
}

1. Introduction. The purpose of this note is to give an elementary demonstration of the validity of the PhragménBrouwer theorem in spaces satisfying certain conditions. This theorem has been proved and generalized by Urysohn and Alexandroff $\dagger$ for cartesian spaces by means of the theory of dimensionality, but the great importance of the theorem seems to the writer to justify the offering of another proof which involves only the elementary principles of the point-aggregate theory. As is well known, to prove this theorem for a space is the same as demonstrating that, if $m$ and $n$ are any two points of the space and $C$ is an irreducible cut between $m$ and $n$, then $C$ is a continuum. It is this form which will be used and the proof is deduced from the validity of the theorem in the euclidean plane.

* Presented to the Society, October 26, 1929. Shortly after the submission of the manuscript of this note to the editors, C. Kuratowski published another proof of this theorem in the Fundamenta Mathematicae, vol. 14, pp. 304-310. This paper was not withdrawn, because the great importance of the theorem seemed to the author to warrant the belief that another demonstration would not be void of interest to readers.

$\dagger \mathrm{P}$. Alexandroff, Sur les multiplicités cantoriennes et le théorème de PhragménBrouwer généralisé, Comptes Rendus, vol. 183, pp. 722-724. 